\title{
Genome-wide scale analyses identify novel BMI genotype-environment interactions using a conditional false discovery rate
}

Moore, R. ${ }^{1,2,3}$, Georgatou-Politou, L. ${ }^{4,5}$, Liley, J., ${ }^{6,7}$ Stegle, O. ${ }^{4,5,2, \#}$, Barroso, I. ${ }^{1,8,9, \#}$

1. Wellcome Sanger Institute, Wellcome Genome Campus, Hinxton, Cambridge, UK, CB10 1SD

2. European Molecular Biology Laboratory, European Bioinformatics Institute, Wellcome Genome Campus, Hinxton, Cambridge, UK, CB10 1SD

3. University of Cambridge, Cambridge, UK

4. Division of Computational Genomics and Systems Genetics, German Cancer Research Center (DKFZ), 69120, Heidelberg, Germany

5. European Molecular Biology Laboratory, Genome Biology Unit, Heidelberg, Germany

6. MRC Biostatistics Unit, University of Cambridge

7. Royal Papworth Hospital, Cambridge

8. MRC epidemiology unit, Institute of Metabolic Science, University of Cambridge, CB2 0SL, UK

9. University of Exeter Medical School, RILD Building, RD\&E Hospital Wonford, Barrack Road, Exeter, EX2 5DW

\# Correspondence should be addressed to Oliver Stegle (o.stegle@dkfz-heidelberg.de) and Inês Barroso (ines.barroso@exeter.ac.uk)

\begin{abstract}
Genotype-environment interaction (GXE) studies typically focus on variants with previously known marginal associations. While such two-step filtering greatly reduces the multiple testing burden, it can miss loci with pronounced $G \times E$ effects, which tend to have weaker marginal associations. To test for $\mathrm{G} \times \mathrm{E}$ effects on a genome-wide scale whilst leveraging information from marginal associations in a flexible manner, we combine the conditional false discovery rate with interaction test results obtained from StructLMM. After validating our approach, we applied this strategy to UK Biobank (UKBB) data to probe for G×E effects on BMI. Using 126,077 UKBB individuals for discovery, we identified known (FTO, MC4R, SEC16B) and novel G×E signals, many of which replicated (FAM150B/ALKAL2, TMEM18, EFR3B, ZNF596-FAM87A, LIN7C$B D N F, F A I M 2, U N C 79, L A T)$ in an independent subset of UKBB $(\mathrm{n}=126,076)$. Finally, when analysing the full UKBB cohort, we identified 140 candidate loci with GXE effects, highlighting the advantages of our approach.
\end{abstract}


Genotype-environment interaction ( $\mathrm{G} \times \mathrm{E})$ studies aim to identify variants with traiteffects that vary under different environmental exposures, which can provide biological insights for understanding trait onset and progression. Additionally, such interactions yield modifiable risk factors, which may be easier to target for disease prevention, and $\mathrm{G} \times \mathrm{E}$ analyses help to identify subgroups of the population at greatest risk of disease who would most benefit from lifestyle or drug intervention for disease prevention ${ }^{1-3}$.

Whilst $G \times E$ effects have already been identified for different traits, including body mass index (BMI) and obesity ${ }^{3-15}$, the number of robustly detected $\mathrm{G} \times \mathrm{E}$ effects remains small ${ }^{2}$.

GXE scans are not currently conducted on a genome-wide scale due to the prohibitive multiple testing burden that they incur, along with lower power to detect interaction effects compared to marginal association effects ${ }^{16,17}$. Instead, $G \times E$ studies are typically based on a two-stage design, where in the first stage variants with marginal association effects are selected and in the second stage, these selected variants are tested for $\mathrm{G} \times \mathrm{E}$ effects. Whilst such independent filtering ${ }^{18}$ reduces the burden of multiple testing, this approach assumes that only variants with sufficiently strong marginal association signals can be subject to interaction effects. Consequently, variants with the strongest and hence most interesting $\mathrm{G} \times \mathrm{E}$ effects may be missed if they fall below the chosen selection threshold for the marginal association signal. This may be particularly problematic if the marginal association signals used are obtained from meta-analyses that combine data across multiple cohorts (in particular for different ancestry groups), which are exposed to different environments. Consequently, there is a need for more flexible approaches that leverage the marginal association signal without the need to perform rigid filtering.

\section{Results}

Here, we combine the conditional false discovery rate (cFDR) with G×E interaction testing using StructLMM ${ }^{10}$ to address the aforementioned limitations of $\mathrm{G} \times \mathrm{E}$ studies. The cFDR has traditionally been applied to leverage the marginal association signal for one trait to improve power in detecting association for a second related trait.

Briefly, the key concept of the cFDR is that an informative covariate allows for estimating and accounting for variations in the distribution of null to non-null variants across all tests ${ }^{19-21}$. The cFDR for a given hypothesis can also be defined as the posterior probability of being null given thresholds on both the $P$ value of the tested hypothesis and the corresponding covariate value ${ }^{19}$.

In this work, we adapt the cFDR to perform genome-wide $\mathrm{G} \times \mathrm{E}$ analysis using the StructLMM interaction test (StructLMM-int) ${ }^{10}$. In this setting, the cFDR allows for leveraging the marginal association signal for the discovery of $\mathrm{G} \times \mathrm{E}$ effects, by performing data driven false discovery. This approach improves the power for $\mathrm{G} \times \mathrm{E}$ analyses, enabling such scans to be conducted at a genome-wide scale by leveraging the information that is contained in the corresponding marginal association signals; 
however, without the need to select arbitrary filtering thresholds. Intuitively, this cFDRbased approach can be interpreted as a 'soft' thresholding equivalent of the commonly employed two-step filtering approach without the need to define rigid thresholds.

\section{Simulated data}

To validate and assess this approach, we initially considered simulated data using 5,000 individuals of European ancestry based on genotypes from the 1000 Genomes Project ${ }^{22}$. Following Moore et al. ${ }^{10}$, we simulated interaction effects based on 60 environmental covariates derived from UKBB. We generated phenotypes with 550 independent genetic effects, with varying fractions of phenotypic variance explained by $\mathrm{G} \times \mathrm{E}$ versus marginal effects ( $\rho$, Methods). For non-zero values of $\rho$ the marginal association signal acts as an informative covariate in assigning the probability that a variant has non-null $\mathrm{G} \times \mathrm{E}$ (Supp. Fig. 1a). Conversely, under the null, when simulating only marginal genetic effects ( $\rho=0$, Supp. Fig. 1b, Methods) or when simulating no causal genetic effects (Supp. Fig. 1c, Methods), the marginal association test results do not act as an informative covariate in assigning the probability that a variant has non-null G×E.

We then assessed the power of the cFDR approach to identify variants with true simulated $\mathrm{G} \times \mathrm{E}$ effects $(\rho>0)$, as well as the power to detect variants within different $\mathrm{G} \times \mathrm{E}$ bins, stratifying based on the strength of the simulated $\mathrm{G} \times \mathrm{E}$ effects $(\rho)$. We compared the results to those obtained from conventional FDR (BenjaminiHochberg ${ }^{23}$ ), as well as two step-filtering for different selection thresholds (Methods). We found that the cFDR approach was better powered than either of these approaches ( $\rho>0,1 \%$ FDR, Fig. 1a); all methods empirically control the FDR (Fig. 1b, Methods). As expected, two-step filtering performs well for identifying variants with weak $\mathrm{G} \times \mathrm{E}$ effects $(\rho<0.5$, Fig. 1a), whereas the conventional FDR when considering all variants is only competitive for detecting variants with very strong $\mathbf{G} \times \mathbf{E}$ effects ( $\rho=1$, Fig. 1a), when the marginal association signal is not informative. In contrast, the cFDR approach performs well in both regimes and in particular overcomes the need to choose a suitable selection threshold for the association signal (conventional FDR corresponds to the $100 \%$ threshold), which will vary depending on the (a priori unknown) extent of $\mathrm{G} \times \mathrm{E}$. 
a)

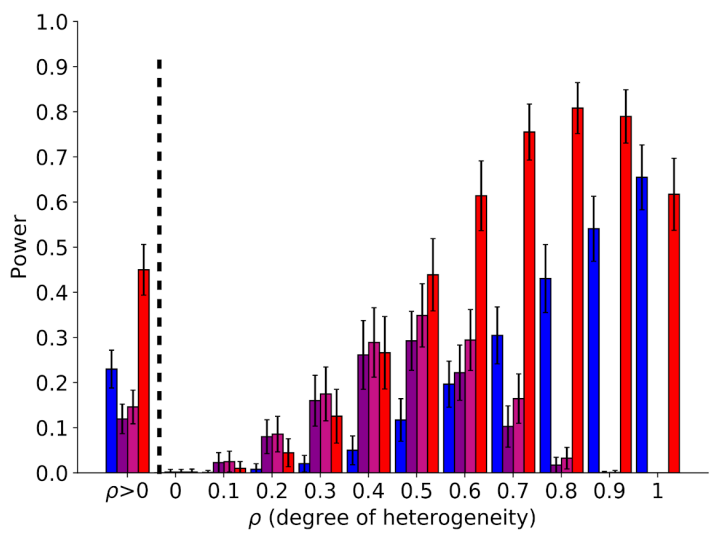

b)

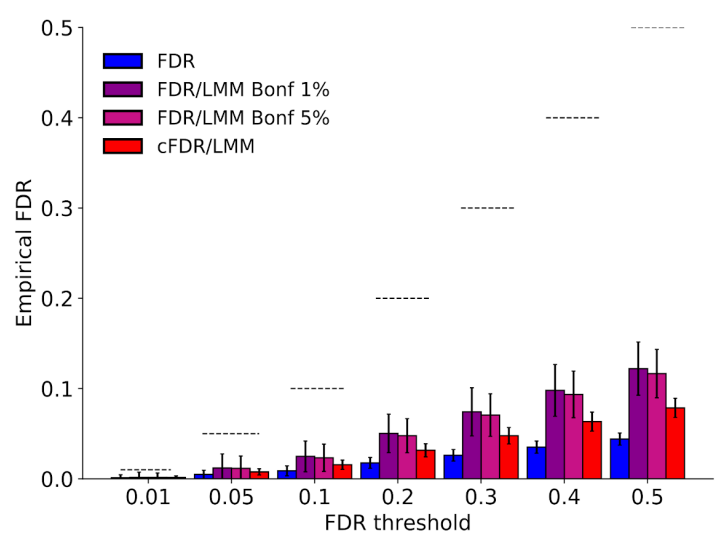

Figure 1 | Assessment of power and empirical FDR control of alternative methods using simulated data. (a) Comparison of power for detecting simulated interaction effects, considering varying fractions of genetic variance explained by $\mathrm{G} \times \mathrm{E}(\rho)$, based on a synthetic European population of 5,000 individuals derived from 1000 Genomes $^{22}$ genotypes (1,650,000 total variants, 550 with non-zero genetic effects; Methods). Shown is power to detect variants with $\mathrm{G} \times \mathrm{E}$ effects (FDR $<1 \%$ ), considering either all variants with $\mathrm{G} \times \mathrm{E}(\rho>0$, left), or variants stratified by the extent of simulated $\mathrm{G} \times \mathrm{E}$ (right). (b) Empirical assessment of the FDR control of alternative methods for increasing thresholds; dashed lines indicate the selected FDR thresholds (Methods). Compared were cFDR considering all 1,650,000 tested variants, FDR (Benjamini-Hochberg ${ }^{23}$ ) considering all $1,650,000$ variants and FDR (Benjamini-Hochberg ${ }^{23}$ ) applied to the subset of variants selected at $1 \%(3,275-5,000$ tested variants per repeat experiment) and 5\% (3,937 - 5,947 tested variants per repeat experiment) FWER based on marginal association effects, using p-values obtained from the StructLMM interaction test and LMM association test for all three methods. Displayed are the average results across 100 repeat experiments, with error bars denoting plus or minus one standard deviation.

\section{Application to data from UK Biobank}

Next, we applied the cFDR approach to UKBB to identify genetic variants with $G \times E$ effects on BMI. We considered 252,153 unrelated UKBB individuals of European ancestry for which BMI and 64 lifestyle environmental factors (diet-related factors, three factors linked to physical activity and six lifestyle factors, modelled as gender and age adjusted, similar to Moore et al. ${ }^{10}$; Methods), were available in the full release of UKBB. We split the cohort randomly into a discovery and validation set consisting of 126,077 and 126,076 individuals, respectively and considered 7,515,856 low-frequency and common variants (imputed variants, MAF > $1 \%$, Methods) for analysis.

On the discovery dataset, we first applied a genome-wide $\mathrm{G} \times \mathrm{E}$ scan using conventional FDR, which identified 78 variants mapping to three loci (ACTBL2-PLK2, FTO, SDCCAG3P1-MC4R) with G $\times$ E effects (Benjamini-Hochberg; FDR $<5 \%$, Methods; Fig. 2a, Supp. Fig. 5a, 6a, 7, Supp. Table 1).

Next, we applied a two-step filtering, whereby 3,767 variants with genome-wide significant BMI association effects $\left(P<5 \times 10^{-8}\right.$, LMM, Supp. Fig. 4) were considered for interaction testing. This identified 330 variants corresponding to eight distinct loci, including two (near FTO and SDCCAG3P1-MC4R) of the three loci identified above, 
with significant $\mathrm{G} \times \mathrm{E}$ effects (Benjamini-Hochberg; FDR $<5 \%$, distinct based on distance clumping of $+/-500 \mathrm{~kb}$ and LD $r^{2}>0.1$, Methods; Fig. 2b, Supp. Fig. 5b, 6b, 8, Supp. Table 1). Notably, because of the assumption that tested variants have substantial marginal effects, by design this approach only identified variants with weak to moderate $\mathbf{G} \times \mathbf{E}$ effects $(\rho<0.556$ for all 330 variants with significant $\mathrm{G} \times \mathrm{E}$ effects, Benjamini-Hochberg FDR $<5 \%$ ).

Finally, we applied cFDR, conditioning the genome-wide $\mathrm{G} \times \mathrm{E}$ test statistics on the corresponding BMI marginal association signal, noting that the marginal association signal is indeed an informative covariate (Supp. Fig. 3). This yielded 964 variants corresponding to 29 loci with significant GXE effects (cFDR $<5 \%,+/-500 k b, r^{2}>0.1$,
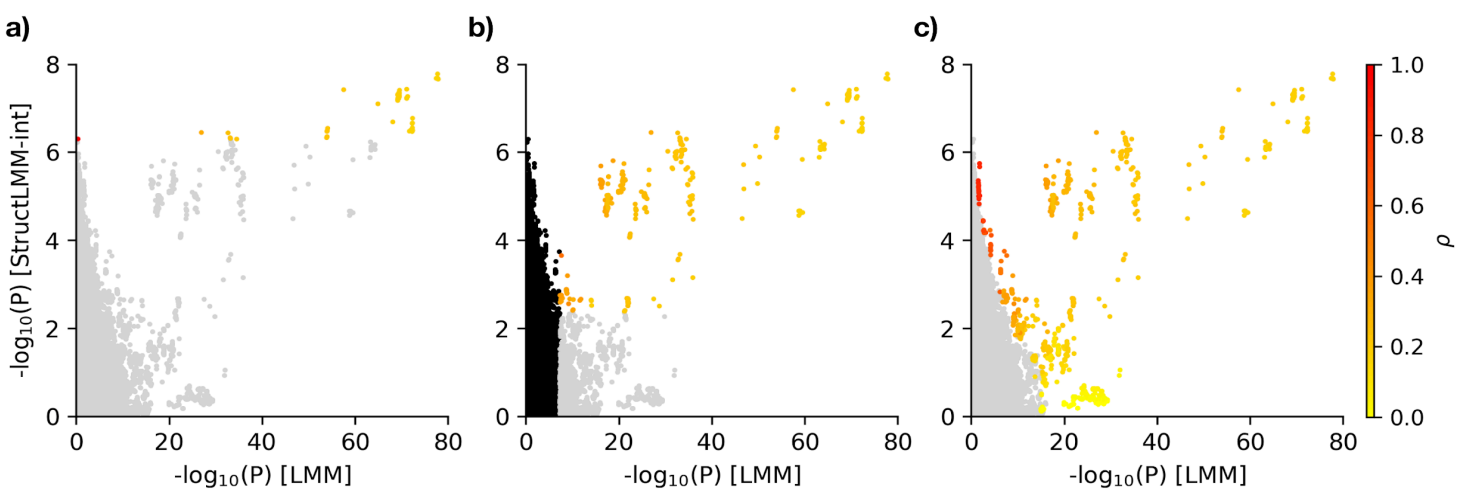

Methods; Fig. 2c, Supp. Fig. 5c, 6c, 9, Supp. Table 1). As well as identifying all loci (and their tag variants) found by the two-step-filtering approach, the cFDR approach identified 21 additional loci, many of which have moderate to strong G×E effects (Fig. 2c, Supp. Fig. 9, Supp. Table 1) with weak to no marginal BMI association effects (Supp. Fig. 5c, Supp. Table 1), clearly highlighting the potential gains of using the cFDR approach.

Figure 2 | Comparison of variants with $\mathbf{G} \times \mathrm{E}$ effects in the discovery set of UKBB identified by alternative methods. Scatter plots of negative log StructLMM interaction $P$ values ( $y$-axis) versus negative log $L M M$ association $P$ values ( $x$-axis, 7,515,856 variants), obtained from UKBB BMI data using the discovery set of individuals $(\mathrm{n}=$ $126,077)$. Variants with $\mathrm{G} \times \mathrm{E}$ effects (FDR < 5\%) are highlighted, with colour denoting the estimated fraction of the genetic variance due to $\mathrm{G} \times \mathrm{E}(\rho)$. Considered were (a) genome-wide analysis where all 7,515,856 variants were tested for interaction effects (78 variants mapping to three loci < 5\% FDR; Benjamini-Hochberg, Methods, Supp. Table 1), (b) two-step filtering considering variants with genome-wide significant marginal association signals ( $P<5 \times 10^{-8} ; 3,767$ variants) for interaction testing (variants in black not tested, 330 variants mapping to eight loci < 5\% FDR; Benjamini-Hochberg Methods, Supp. Table 1) and (c) a genome-wide analysis of all 7,515,856 variants using cFDR (964 variants mapping to 29 loci < 5\% cFDR, Methods, Supp. Table 1). Loci were defined using LD clumping $\left(r^{2}<0.1,+/-500 \mathrm{~kb}\right.$; Methods).

To further assess the additional associations identified, we assessed the replication of the variants detected by the cFDR approach for replication in the independent validation set of individuals $(n=126,076)$, again using the StructLMM interaction test.

Globally, we observed that variants with $\mathrm{G} \times \mathrm{E}$ identified in the discovery set were enriched for small $p$-values in the validation set, which includes the 21 loci that were 
exclusively identified by the cFDR (Fig. 3a, Supp. Fig. 10). Consistent with this, the tail strength $^{24}$ (deviation measure between the observed and expected set of $p$-values) for the 29 cFDR loci exceeded the chance expectation (empirical $P<0.002$ based on 500 matched sets of null variants ${ }^{25}$, Methods; $P<0.002$ for the 21 loci), which is similar to loci identified by the two-step filtering approach $(P<0.002)$.

In addition, the lead variant is nominally significant $(P<0.05)$ in the validation dataset for eight (SEC16B, ALKAL2-TMEM18, ZNF596-FAM87A, FAIM2, LAT, FTO, SDCCAG3P1-MC4R and POFUT2) of the 29 discovered loci, which exceeds the chance expectation (empirical $\mathrm{P}<0.002$, using 500 matched sets of null variants, Methods). In comparison, only three (FTO, SDCCAG3P1-MC4R and LAT) of eight loci for the twostep filtering approach (empirical $P=0.012$, using 500 matched sets of null variants, Methods) and two (FTO, SDCCAG3P1-MC4R) of three loci for the genome-wide FDR approach (empirical $P=0.01$, using 500 matched sets of null variants, Methods) are nominally significant $(P<0.05)$ in the validation dataset. Considering the 21 loci unique to the cFDR approach in discovery, the lead variant is nominally significant $(P<0.05)$ for five (SEC16B, ALKAL2-TMEM18, ZNF596-FAM87A, FAIM2 and POFUT2) of the 21 loci (empirical $P=0.002$, using 500 sets of matched variants, Methods). These results indicate that the rate of replication of variants discovered using the cFDR approach is as good as using a conventional two-step filtering pipeline.

a)

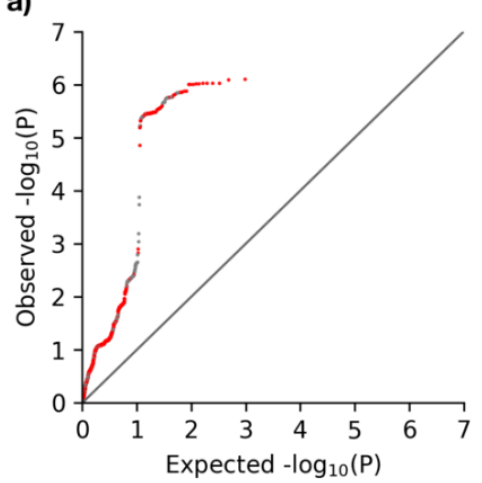

b)

\begin{tabular}{|c|c|c|c|c|c|c|c|}
\hline & $\begin{array}{c}\text { Number of } \\
\text { variants } \\
\text { identified } \\
\text { in } \\
\text { discovery }\end{array}$ & $\begin{array}{c}\text { Number } \\
\text { of loci } \\
\text { identified } \\
\text { in } \\
\text { discovery }\end{array}$ & $\begin{array}{c}\text { Tail } \\
\text { strength } \\
\text { empirical } \\
\text { P value }\end{array}$ & $\begin{array}{c}\text { Number of } \\
\text { nominally } \\
\text { significant } \\
\text { loci }\end{array}$ & $\begin{array}{c}\text { Nominally } \\
\text { significant } \\
\text { empirical } \\
\text { P value }\end{array}$ & $\begin{array}{c}\text { Number of } \\
\text { variants } \\
\text { that } \\
\text { validate at } \\
\text { 5\% FDR } \\
\text { (Storey) }\end{array}$ & $\begin{array}{c}\text { Number } \\
\text { of loci } \\
\text { that } \\
\text { validate } \\
\text { at 5\% } \\
\text { FDR } \\
\text { (Storey) }\end{array}$ \\
\hline cFDR & 964 & 29 & $<0.002$ & 8 & $<0.002$ & 611 & 16 \\
\hline cFDR only & 634 & 21 & $<0.002$ & 5 & 0.002 & 390 & 11 \\
\hline $\begin{array}{c}\text { Two-step filtering } \\
\text { FDR }\end{array}$ & 330 & 8 & $<0.002$ & 3 & 0.012 & 222 & 5 \\
\hline $\begin{array}{c}\text { Genome-wide } \\
\text { FDR }\end{array}$ & 78 & 3 & - & 2 & 0.01 & 73 & 2 \\
\hline
\end{tabular}

Figure 3 | Replication of variants with GxE in the validation datasets. (a) QQ plot of negative $\log P$ values from the StructLMM interaction test evaluated in the validation dataset $(n=126,076)$ for the 964 variants identified by the cFDR approach in the discovery analysis. The 330 variants also identified by the genome-wide FDR and/or the two-step filtering in the discovery set are displayed in grey whilst the 634 variants exclusively identified by the cFDR approach are displayed in red. (b) Table summarising the validation metrics considered. Columns 1 and 2 state the number of variants and loci (LD clumped loci, $r^{2}<0.1$ within $+/-500 \mathrm{~kb}$ ), respectively with significant $\mathrm{G} \times \mathrm{E}$ effects (FDR $5 \%$ ) identified in the discovery set of UKBB individuals $(n=126,077)$; column 3 displays the significance of the tail strength of the replication $P$ values (based on 500 sets of matched null variants; Methods); column 4 states the number of loci identified in discovery that are nominally significant (StructLMM interaction $\mathrm{P}<0.05$ ) in validation set, column 5 the corresponding empirical $P$ value based on 500 sets of matched variants (Methods), columns 6 and 7 the number of variants that validate the number of variants and loci respectively that validate (Storey FDR $5 \%{ }^{26,27}$ ). 'cFDR only' results are based on variants/loci that were only found using the cFDR multiple testing correction. 
Even higher numbers of loci replicated when using Storey's $Q$ values (estimates the fraction of non-null variants and does not assume it is 1 as for the Benjamini-Hochberg approach) ${ }^{26,27}$, providing further support for using the cFDR when testing for G×E. Sixteen of the loci identified with the cFDR approach replicate (FDR $<5 \%$ ), whilst in comparison, only five and two of the loci identified by the conventional filtering approach and the genome-wide FDR approach replicate (both are a subset of those that replicate based on the cFDR approach), such that ten Ioci (SEC16B, ALKAL2-TMEM18 two distinct signals, ZNF596-FAM87A, SEM4AD-GADD456, ITGB1-NRP1, FAIM2, SDCCAG3P1-MC4R two distinct signals, POFUT2) are identified and replicate solely through the use of the cFDR (Supp. Table 1). BDNF is identified both by cFDR and the two-step filtering approach but only replicates in the cFDR approach (Supp. Table 1).

Finally, having validated the approach, we took advantage of the full set of 252,188 individuals in UKBB, to increase power to identify additional candidate loci with $\mathrm{G} \times \mathrm{E}$ effects on BMI. The cFDR approach identified 140 loci with significant GXE effects (cFDR $<5 \%,+/-500 k b, r^{2}>0.1$, Methods, Supp. Fig. 11 c, f, Supp. Fig. 13c, Supp. Table 2). In comparison, the genome-wide and conventional two-step filtering approaches identified six and 23 loci with significant GXE effects (FDR < 5\%, +/-500kb, $r^{2}>0.1$, Methods, Supp. Fig. 11, Supp. Fig. 12, Supp. Fig. 13, Supp. Table 2), respectively.

Among the 140 loci identified from the full UKBB dataset, in addition to loci overlapping those identified in the discovery and validation datasets, several others merit additional validation in other large biobanks. Of particular interest are ten loci where marginal association with $B M I$ is weak $\left(P>10^{-3}\right)$ but with strong evidence of $G \times E\left(P<10^{-5}\right)$ and where $\rho$ is large (>0.5) (Supp. Table 2). Among these is CADM2, a locus previously associated with BMI, obesity and adiposity traits ${ }^{28-32}$, in addition to reported associations with physical activity ${ }^{33}$, risk taking behaviour, alcohol, smoking and many other behavioural and cognitive traits ${ }^{34}$, it has recently been suggested to form a link between psychological traits and obesity ${ }^{35}$.

\section{Discussion}

To date, $\mathrm{G} \times \mathrm{E}$ discovery efforts have lagged behind genetic association studies due to limitations in available methods, and sample sizes. Genome-wide $\mathrm{G} \times \mathrm{E}$ scans have a high multiple-testing cost and are therefore limited in power. In contrast, two-step filtering approaches whereby only genetic variants with prior evidence of marginal association with a given trait or disease of interest are tested for $\mathrm{G} \times \mathrm{E}$ effect, limit the multiple-testing burden but are constrained by the number of variants tested and the need to set arbitrary association significance thresholds for filtering. To overcome these limitations, we describe the use of the conditional false-discovery (cFDR) approach, aligned with our recently described StructLMM interaction test method ${ }^{10}$, to perform a genome-wide search for $\mathrm{G} \times \mathrm{E}$ effects whilst avoiding the high multiple testing burden.

Using both simulated data and UKBB data to identify novel loci with evidence of GXE effects on BMI, we highlight two significant advantages of the cFDR approach compared to genome-wide FDR, or two-step filtering approaches. Firstly, cFDR allows a genome- 
wide $\mathrm{G} \times \mathrm{E}$ exploration whilst retaining greater discovery power compared to conventional genome-wide FDR. Secondly, variants found uniquely by cFDR validate, in an independent dataset, at an equivalent rate to those identified by the conventional hardfiltering approach, demonstrating the utility of the approach to discover variants with a previously unsuspected effect on the trait in question. We further demonstrate that focusing on variants with marginal associations may paradoxically limit the ability to discover variants with the highest $\mathrm{G} \times \mathrm{E}$ effects, because many of these will either have been filtered out in large meta-analyses due to large heterogeneity between contributing datasets, or they will have insufficient evidence for trait-association in combined datasets to be taken forward for $\mathrm{G} \times \mathrm{E}$ testing. This is demonstrated by the two-step filtering approach only identifying loci with low $\rho$ value, whereas those loci identified by the cFDR approach have a greater distribution of $\rho$ values (Fig. 2, Supp. Fig. 11).

Using the cFDR approach in combination with StructLMM, we detected 16 replicating loci (only five of which were identified and validated with a conventional two stage design) with evidence of $\mathrm{G} \times \mathrm{E}$ effects on BMI. Two (FTO and MC4R), had been previously established $^{5,7,9,10,15,36}$, and were also detected when applying genome-wide FDR or twostep filtering approaches. In addition, for the first time we demonstrate evidence of $\mathrm{G} \times \mathrm{E}$ effects on BMI, in EFR3B, LINC7C and UNC79, replicating loci overlapping between cFDR and two-step filtering approaches.

Furthermore, eleven replicating loci were uniquely identified by the cFDR approach (Supp. Table 1). Amongst these eleven loci, is $S E C 16 B$, a positive control for which previous secondary analyses provided some evidence for an interaction $(P=0.025)$ with physical activity in Europeans ${ }^{36}$, and in Hispanics ${ }^{37}$ and identified more recently by us using the StructLMM interaction test with a sample size approximately twice as large, though we note it contains the samples used here ${ }^{10}$. Notable loci with new evidence for GXE effects on BMI include ALKAL2, TMEM18 and FAIM2. ALKAL2 and FAIM2 were also detected when using a two-step filtering approach but only when the entire UKBB dataset was used. ALKAL2 (previously called FAM150B) lead variant rs62107263 $(M A F=22 \%)$ is in $D^{\prime}=1$ with rs62107261 $(M A F=4 \%)$, previously associated with obesity and body fat distribution ${ }^{32,38}$, lung function ${ }^{39}$ and smoking status ${ }^{39}$. In TMEM18 the variant with $\mathrm{G} \times \mathrm{E}$ effects (rs74676797) is in perfect LD $\left(r^{2}=1\right)$ with $r 13021737$ previously shown to associate with BMI and obesity ${ }^{29,40,41}$. Finally, in FAIM2 (rs7132908), the same variant has been previously associated with childhood obesity ${ }^{41}$, severe obesity ${ }^{32}, \mathrm{BMI}^{39}$ and alcohol consumption ${ }^{42}$. The latter is interesting as the environment with the most evidence of driving the interaction effect is alcohol frequency in women (Supp. Fig. 14).

In summary, we describe a novel approach that uses the cFDR to discover GXE effects genome-wide without incurring the high multiple testing burden. While we describe an application that combined the cFDR approach with our previous StructLMM interaction test ${ }^{10}$, it is important to note that the cFDR approach can be combined with any G×E test and therefore has wide applicability. Additionally, we note that while we considered marginal association results with BMI as our informative covariate, it is equally valid to use marginal association results with one trait to test for $G \times E$ interactions on a second trait, for example, we could use marginal associations with $B M I$, to look for $G \times E$ on type 2 diabetes risk. 


\section{Online methods}

\section{Statistical tests}

\section{LMM}

A conventional linear mixed model (LMM) to test for marginal associations can be cast as

$\boldsymbol{y}=\boldsymbol{X} \boldsymbol{b}+\boldsymbol{x} \beta+\boldsymbol{u}+\boldsymbol{\psi}$

where $\beta$ is the effect size of the focal variant $x, X$ denotes the fixed-effect design matrix of $K$ covariates and $\boldsymbol{b}$ the corresponding effect sizes. The variable $\boldsymbol{u}$ denotes additive (confounding) factors, and $\boldsymbol{\psi}$ denotes i.i.d. noise. The random effect component $\boldsymbol{u}$ and the noise vector $\boldsymbol{\psi}$ follow multivariate normal distributions, $\boldsymbol{u} \sim N\left(\mathbf{0}, \sigma_{e}^{2} \boldsymbol{\Sigma}\right)$ and $\boldsymbol{\psi} \sim N\left(\mathbf{0}, \sigma_{n}^{2} \mathbf{I}\right)$, where the covariance matrix $\boldsymbol{\Sigma}$ reflects the covariance of environment across individuals. $\boldsymbol{\Sigma}$ is estimated using a linear covariance function such that $\boldsymbol{\Sigma}=\mathbf{E E}^{T}$, where $\mathbf{E}$ is an $N \times L$ matrix composed of $L$ environmental exposures for each of the $N$ individuals (see Moore et al. ${ }^{10}$ for full details). Marginal association tests for non-zero effects of the focal variant correspond to alternative hypothesis $\beta \neq 0$.

\section{StructLMM interaction test}

The StructLMM interaction test generalises the LMM by introducing per-individual effect sizes of the focal variant due to $\mathrm{G} \times \mathrm{E}$ and can be cast as

$\boldsymbol{y}=\boldsymbol{X} \boldsymbol{b}+\boldsymbol{x} \beta+\boldsymbol{x} \odot \boldsymbol{\beta}_{\mathrm{G} \times \mathrm{E}}+\boldsymbol{u}+\boldsymbol{\psi}$

where $\odot$ denotes the Hadamard product and $\boldsymbol{\beta}_{\mathrm{G} \times \mathrm{E}}$ is a per-individual allelic effects vector and follows a multivariate normal distribution with environment covariance $\boldsymbol{\Sigma}$ : $\boldsymbol{\beta}_{\mathrm{G} \times \mathrm{E}} \sim N\left(\mathbf{0}, \sigma_{\mathrm{G} \times \mathrm{E}}^{2} \boldsymbol{\Sigma}\right)$, where $\boldsymbol{\Sigma}=\mathbf{E E}^{T}$ as described above. As a result the interaction test, with alternative hypothesis $\sigma_{\mathrm{G} \times \mathrm{E}}^{2}>0$, tests for $\mathrm{G} \times \mathrm{E}$ effects due to potentially multiple environmental variables (see Moore et al. ${ }^{10}$ for full details). It can be seen that under the null StructLMM reduces to the LMM described above.

\section{Fraction of genetic variance explained by $\mathrm{G} \times \mathrm{E}(\rho)$}

In this study, $\rho=\frac{\operatorname{Var}^{G \times E}}{\operatorname{Var}^{G}+\operatorname{Var}^{G \times E}}$ is defined as the fraction of genetic variance that is explained by $G \times E$, where $\operatorname{Var}^{G}$ denotes the fraction of the variance explained by marginal genetic effects and $\operatorname{Var}{ }^{\mathrm{G} \times \mathrm{E}}$ variance due to $\mathrm{G} \times \mathrm{E}$. An estimate of $\rho$ can be obtained by maximizing the marginal likelihood of Eq. (2) (see Moore et al. ${ }^{10}$ for details).

\section{The conditional false discovery rate (cFDR)}

The cFDR is an estimate of the posterior probability of a primary trait of non-GXE interaction given $p$-value thresholds for hypothesis tests of $G \times E$ interaction and for a secondary trait of phenotype-genotype $(G)$ association. In this work, p-values for the 'primary trait' are obtained from application of the StructLMM interaction (StructLMMint) test (see above) and the conditional informative covariate trait as the association test results, obtained from LMM (see above, Moore et al. ${ }^{10}$ description of LMM-Renv for full details). 


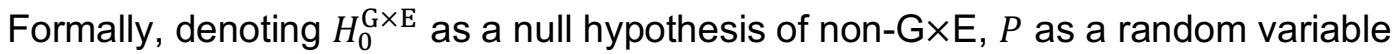
corresponding to the $p$-value from a hypothesis test for $G \times E$ from StructLMM, and $Q$ as a $p$-value from a hypothesis test for phenotype association, the cFDR is a function of thresholds $p, q$ :

$c F D R(p, q)=P\left(H_{0}^{\mathrm{G} \times \mathrm{E}} \mid P \leq p, Q \leq q\right)$

Given observed $(P, Q)$ values $\left(p_{1}, q_{1}\right),\left(p_{2}, q_{2}\right), \ldots,\left(p_{n}, q_{n}\right)$ for each SNP, ranking hypothesis by $\operatorname{cFDR}\left(p_{1}, q_{1}\right), c F D R\left(p_{2}, q_{2}\right), \ldots, c F D R\left(p_{n}, q_{n}\right)$ may sort null and non-null SNPs more effectively than ranking by $\mathrm{p}$-values $p_{1}, p_{2}, \ldots, p_{n}$ alone. The best possible metric by which to rank SNPs can be shown ${ }^{19}$ to be:

$P\left(H_{0}^{\mathrm{G} \times \mathrm{E}} \mid P=p, Q=q\right)$

but this quantity is difficult to estimate, whereas the cFDR is readily and partlyconsistently estimable from empirical cumulative density function of $P$ and $Q$.

The cFDR can be seen to be related to the Benjamini-Hochberg procedure. The Benjamini-Hochberg procedure controlling FDR at $\alpha$ is roughly equivalent to rejecting the null for all $i$ such that:

estimated $\left\{P\left(H_{0}^{\mathrm{G} \times \mathrm{E}} \mid P \leq p_{i}\right)\right\}<\alpha$

A corresponding rule for the cFDR does not hold ${ }^{21}$; rejecting $H_{0}^{\mathrm{G} \times \mathrm{E}}$ whenever estimated $\{c F D R(p, q)\}<\alpha$ does not control the FDR at $\alpha$.

We control type-1 error rate (as FDR) using an approach proposed by Liley et al. ${ }^{19}$ (code available at https://github.com/jamesliley/cfdr, using options mode $=2$ and adj = T). Briefly, in this method, type-1 error rate (as FDR) is controlled by using the estimated cFDR to define a map from the unit square (the domain of $P, Q$ )) to the real line. This enables 'contours' of cFDR to be drawn through each $p$-value pair of interest and the regions within integrated with respect to an estimated distribution of the $p$ value pairs under $H_{0}^{\mathrm{G} \times \mathrm{E}}$, thereby transforming the set of pairs of $\mathrm{p}$-values into a set of single $\mathrm{p}$-values against $H_{0}^{\mathrm{G} \times \mathrm{E}}$ which encompass the additional information from the covariate (and can be used in the Benjamini-Hochberg procedure to control FDR). For correct calibration of the resultant $p$-value, the map must be independent of the $p$-value pairs it is used on, so in each case we fitted the map using only $p$-values from SNPs in linkage equilibrium with the SNP under investigation.

Both the cFDR and two-stage independent filtering ${ }^{18}$ assume independence of $P$ and $Q$ under $H_{0}^{\mathrm{G} \times \mathrm{E}}$ (although both may be adapted if this condition does not hold, and cFDR estimates are reasonably robust to small correlations under the $H_{0}^{\mathrm{G} \times \mathrm{E} 21}$ ). It can be shown that independence of $P$ and $Q$ holds for nested models (StructLMM interaction test reduces to LMM under the null as described above $)^{43}$. This is in agreement with our simulation experiments that indicate results from the StructLMM interaction test are independent of those from the LMM association test (Supp. Fig. 1).

\section{Simulations}

\section{Simulation procedure overview}


Simulations were based on genotypes of European individuals from the 1000 Genomes project ${ }^{22}$ (phase 1, 1,092 individuals, 379 Europeans), considering 103,527 variants on chromosome 21 (minor allele frequency $\geq 2 \%$ ). Following ${ }^{10,44,45}, 5,000$ synthetic genotypes of unrelated individuals were generated for different sample sizes, while preserving the population structure of the seed population $\left(\mathrm{see}^{46}\right)$. We considered 33 environmental exposures using empirical environmental covariates from 70,282 UKBB individuals (based on the Interim release), augmented with gender (binary male indicator vector and binary female indicator vector) and age (continuous vector), resulting in 99 lifestyle covariates and age itself, giving a total of 100 environmental variables. 60 of these environments were selected at random with a subset of 30 environments used to simulate $\mathrm{G} \times \mathrm{E}$ effects and all 60 environments used for testing. These environmental variables were preprocessed as in the UKBB analysis (see below) and randomly assigned to synthetic genotypes (see Moore et al. ${ }^{10}$ for full details).

We randomly select 550 segments of approximately $2 \mathrm{Mb}$ from chromosome 21 , simulating genetic effects from one causal variant. 50 of these segments were simulated with marginal genetic effects only $(\rho=0)$ and $\mathrm{G} \times \mathrm{E}$ effects of varying strengths were simulated for the remaining 500 segments $(\rho=0.1,0.2,0.3,0.4,0.5$, $0.6,0.7,0.8,0.9$ and 1.0 for 50 segments each). This procedure is repeated 100 times; thereby generating 100 phenotypes, with each the sum of 50 marginal association effects and $500 \mathrm{G} \times \mathrm{E}$ effects.

\section{Conditional QQ plots}

$Q Q$ plots based on one of the repeat experiments of the StructLMM interaction results were generated for the subset of variants with LMM association p-values $<1$ (blue),$<5$ $\times 10^{-3}$ (orange), $<5 \times 10^{-5}$ (green) and $<5 \times 10^{-8}$ (purple), referred to as conditional QQ plot (Supp. Fig. 1a). For comparison, we simulated phenotypes with no dependence between the association and interaction test results, considering i) simulated marginal genetic effects (550 variants with marginal genetic effects but no G×E effects $(\rho=0)$, Supp. Fig. 1b) and ii) no simulated genetic effect (Supp. Fig. 1c).

\section{Assessment of the discovery rate control using simulations}

If a variant that lies within a block simulated to have only marginal genetic effects $(\rho=$ 0 ) was declared as having a significant interaction effect, this was defined as a false discovery whilst if a variant lies within a block with simulated causal $\mathrm{G} \times \mathrm{E}(\rho>0)$ was declared as having a significant interaction effect, this was defined as a true discovery.

The empirical FDR was then calculated as the number of $\frac{\text { false discoveries }}{\text { true discoveries+false discoveries, }}$, with the average value and standard error over the 100 repeat experiments calculated (Fig. 1b).

\section{Power simulations}

We first calculated power to detect any GxE effects $(\rho>0)$ and then power to detect the subset of simulated causal variants for each of the 11 values of $\rho$. Power was assessed at the $1 \%$ FDR, considering variants in linkage disequilibrium with the selected true causal variants $\left(\mathrm{r}^{2} \geq 0.8\right)$ as true positives, reporting the average power and standard error across the 100 repeat experiments (each experiment has power 0 , 
$0.002,0.004, \ldots, 0.996,0.998,1$ when considering power to detect any interaction effect and $0,0.02,0.04 \ldots, 0.96,0.98,1$ when considering power to detect variants at each value of $\rho$, Fig. 1a).

\section{Comparison methods}

We compared the cFDR to alternative multiple test strategies. We considered testing all variants for interaction effects at $1 \%$ FDR (Benjamini-Hochberg). We also considered two-stage independent filtering approaches ${ }^{18}$, in which variants were selected for interaction testing based on their association results (LMM) at $1 \%$ and $5 \%$ FWER. For any of the two-stage filtering approaches, a 1\% FDR (Benjamini-Hochberg) was then applied to the subset of variants that were tested for interaction effects.

\section{Analysis of BMI in UK Biobank}

This research has been conducted using the full release of the UK Biobank Resource (Application 14069) $^{47}$. The UK Biobank study has approval from the North West MultiCentre Research Ethics Committee and all participants included at the time of the analyses provided informed consent to UK Biobank.

\section{Data Preprocessing}

The data was preprocessed as described in Moore et al. ${ }^{10}$. Briefly, BMI phenotype data is 'Instance 0' of UKBB data field 21001. Individuals with missing BMI data were discarded and BMI log transformed ${ }^{15,48}$. We considered 21 lifestyle covariates as environments, discarding individuals with outlying or missing environmental data (see Supp. Note in Moore et al. ${ }^{10}$ for details). We further discarded individuals of non-British ancestry, related individuals and those that had withdrawn consent at the time of analysis. After these QC procedures on the BMI phenotype, genotype and environmental variables, we had a set of 252,153 individuals for analysis. These individuals were randomly split into a discovery set $(n=126,077)$ and a validation set $(n=126,076)$. Principal components for population structure were those computed by Moore et al. ${ }^{10}$, using flashPCA version $2.0^{49}$ using 147,604 variants as indicated by the field 'in_PCA' from the released marker $Q C$ file.

\section{Genotype data}

The genetic variants considered were the same as those used in Moore et al. ${ }^{10}$. Specifically, 7,515,856 variants imputed with the HRC panel (build GRCh37) with missingness $<5 \%$, MAF $>1 \%$, HWE P $>1 \times 10^{-6}$, and INFO score $r^{2}>0.4$ were considered (see Moore et al. ${ }^{10}$ for full details).

\section{Environmental covariance and covariates}

The environmental matrices $\mathbf{E}$ for the discovery, validation and analysis using all available samples were generated by augmenting the 21 environmental variables described above with gender (binary male indicator vector and binary female indicator vector) and age (continuous vector), resulting in 63 covariates and age itself was also included, giving a total of 64 environmental factors (see Supp. Note in Moore et al. ${ }^{10}$ for details). The environmental covariance was then estimated using standardised environmental variables followed by per-individual standardisation (see Supp. Note in 
Moore et al. ${ }^{10}$ for details). In all analyses, a mean vector, genotype chip, gender, age ${ }^{2}$, age $^{3}$, gender $\times$ age, gender $\times$ age $^{2}$, gender $\times$ age $^{3}$ and ten genetic principal components were included as covariates.

\section{Calibration of the considered tests}

To check that the association and interaction tests were calibrated under the null, we assessed the empirical calibration using permuted genotype variants $(89,166$ variants) on chromosome 22 (Supp. Fig. 2). Genomic control was calculated as $\lambda_{G C}=\frac{\log _{10}(m)}{\log _{10}(0.5)}$; $m$ is the median StructLMM interaction $P$ value.

\section{Discovery interaction testing}

In these analyses we used only the discovery set of 126,077 individuals.

For the genome-wide FDR approach we applied the 5\% Benjamini-Hochberg FDR ${ }^{23}$ to the corresponding $\mathrm{G} \times \mathrm{E}$ results obtained using StructLMM interaction test for all $7,515,856$ that passed $Q C$, identifying 78 variants with significant $\mathrm{G} \times \mathrm{E}$ effects.

For the conventional two-step filtering FDR approach, we selected the 3,767 variants with association $\mathrm{P}<5 \times 10^{-8}$ and then applied the $5 \%$ Benjamini-Hochberg FDR $^{23}$ to the corresponding $\mathrm{G} \times \mathrm{E}$ results obtained using StructLMM interaction test, identifying 330 variants with significant $\mathrm{G} \times \mathrm{E}$ effects.

For the cFDR approach we applied the $5 \%$ cFDR procedure (described above ${ }^{19}$ ), using 238 folds containing between 30,025 and 44,583 consecutive variants and excluding a fold either side of the focal fold such that the minimum distance between a focal SNP in fold and a SNP out of fold used for the inference was greater than 5,329,663 bp and the maximum LD (based on 100 boundary variants) $r^{2}<9.50 \times 10^{-4}$ and to the $\mathrm{G} \times \mathrm{E}$ results obtained using StructLMM interaction test for all 7,515,856 that passed QC, using the corresponding association results obtained from LMM for all 7,515,856 variants as the informative covariate, identifying 964 variants with significant $\mathrm{G} \times \mathrm{E}$ effects.

For all variants with significant $\mathrm{G} \times \mathrm{E}$ effects, we estimated $\rho$, which is the fraction of the genetic variance explained by $\mathrm{G} \times \mathrm{E}$ effects (see Moore et al. ${ }^{10}$ for details). A value of $\rho$ close to 0 , means that the effect of variant is largely the same across all individuals with only a small component of the genetic effect dependent on environmental exposures whilst a value close to 1 means that the effect of variant is dependent on the environmental exposures.

LD clumping was performed for each method to identify independent loci by: iteratively (i) selecting the most significant variant (based on the StructLMM interaction results), (ii) removing all variants in LD $\left(r^{2}>0.1\right.$ based on the UKBB discovery set of individuals) within $+/-500 \mathrm{~kb}$ until no variants were left resulting in three, eight, and 29 clumps (loci) respectively (Supp. Table 1). Variants in each loci are visualised in Supp. Figs. 7, 8 and $\mathbf{9}$, with variants in the clump coloured according to $\rho$ and the lead variant is represented by a diamond. 


\section{Validation}

In these analyses we used only the validation set of 126,076 individuals.

We first tested the 964 variants identified with significant G×E effects (cFDR $<5 \%$ ) in the discovery analysis for interaction effects using the StructLMM interaction test considering the validation set of individuals. $Q Q$ plots of the validation StructLMM interaction test results were plotted where (i) the 330 variants also identified by the genome-wide FDR and/or the conventional two-step filtering approach using the discovery set of individuals were coloured in grey (634 variants in red were identified only with the cFDR approach, Fig. 3) and (ii) all variants (615 variants) within the eight loci also identified by the genome-wide FDR and/or the conventional two-step filtering approach using the discovery set of individuals were coloured in grey (340 variants in the 21 loci identified only by the cFDR approach are shown in red, Supp. Fig. 10).

We assessed the deviation from the null distribution using the tail strength measure ${ }^{24}$ using the lead variant for each locus identified in the discovery analysis based on all 29 cFDR loci, the 8 loci identified by the two-step filtering approach and for the 21 loci identified only by the cFDR approach. Empirical $P$ values were calculated by identifying 500 different matched variants for each lead variant using SNPsnap ${ }^{25}$ (https://data.broadinstitute.org/mpg/snpsnap/match snps.html), using default settings apart from setting the 'Number of matched SNPs' to 4000) and then calculating tail strength for each matched set and subsequently the fraction of the 500 matched sets that are at least as extreme as the observed tail strength measure.

We also counted the number of loci with nominally significant $P$ values (StructLMM interaction test in validation $<0.05$ ) identified by each approach in discovery and also for the 21 loci only identified by the cFDR approach based on lead variants per locus identified in the discovery analysis. Empirical $P$ values were calculated using the same sets of matched variants as were used for the tail strength measure.

Finally, we identified loci that validated for each method at the 5\% FDR using Storey's $q$ value approach ${ }^{26,27}$. This involves estimating the proportion of tested variants that are truly null $\left(\pi_{0}\right)$ based on the set of variants tested. As the number of variants identified in discovery for the genome-wide FDR and two-step filtering approaches is too low to estimate $\pi_{0}$, the estimate of $\pi_{0}$ obtained for the cFDR approach $(0.202$, https://www.bioconductor.org/packages/devel/bioc/vignettes/qvalue/inst/doc/qvalue.pdf ) was used.

\section{Interaction testing using all samples}

The data for this analysis was preprocessed as described above but at the time of analysis less individuals had withdrawn consent, resulting in a total of 252,188 individuals. The analysis was conducted analogous to that described in the discovery interaction testing methods section, with the only difference being the larger sample size.

\section{Explorative analysis of driving environments at rs7132908}

As described in Moore et al. ${ }^{10}$, we explored which environments had putative effects on $\mathrm{G} \times \mathrm{E}$ by comparing the log marginal likelihood of the full model to models with 
individual or sets of environments excluded. We initially assessed the relevance of individual environments based on the log(Bayes factor) of removing single environments (Supp. Fig. 14a). To account for correlations between environments, we also used a backwards elimination procedure (see Moore et al. ${ }^{10}$ Supp. Note for full details), greedily removing environments until there is evidence that we have selected a full set of environments that can drive the observed GXE effect (Supp. Fig. 14b).

\section{Acknowledgements}

This research has been conducted using the UK Biobank Resource (Application Number 14069). R.M. was supported by a PhD fellowship from the Mathematical Genomics and Medicine programme, funded by the Wellcome Trust. IB acknowledges funding from Wellcome (WT206194). J.L. was funded by Johnson and Johnson. O.S. is supported by the BMBF, the European Commission (ERC project DECODE, grant agreement ID: 810296) and the Volkswagen Foundation. L.G.P. was supported by core funding.

\section{Author contributions}

R.M, L.G.P., I.B. \& O.S. designed the experiments. R.M. \& L.G.P. analysed the simulation data. R.M. analysed the UK Biobank data. J.L. provided code and advice for the cFDR analysis. R.M., L.G.P., I.B. \& O.S. interpreted results and wrote the manuscript. All authors read and approved the final version of the manuscript.

\section{Competing interests}

R.M. is now employed by Genomics plc

\section{References}

1. Hunter, D.J. Gene-environment interactions in human diseases. Nat Rev Genet 6, 287-98 (2005).

2. McAllister, K. et al. Current Challenges and New Opportunities for GeneEnvironment Interaction Studies of Complex Diseases. Am J Epidemiol 186, 753-761 (2017).

3. Rask-Andersen, M., Karlsson, T., Ek, W.E. \& Johansson, A. Gene-environment interaction study for BMI reveals interactions between genetic factors and physical activity, alcohol consumption and socioeconomic status. PLoS Genet 13, e1006977 (2017).

4. Ahmad, T. et al. Lifestyle interaction with fat mass and obesity-associated (FTO) genotype and risk of obesity in apparently healthy U.S. women. Diabetes Care 34, 675-80 (2011).

5. Bjornland, T., Langaas, M., Grill, V. \& Mostad, I.L. Assessing gene-environment interaction effects of FTO, MC4R and lifestyle factors on obesity using an extreme phenotype sampling design: Results from the HUNT study. PLoS One 12, e0175071 (2017).

6. Corella, D. et al. A high intake of saturated fatty acids strengthens the association between the fat mass and obesity-associated gene and BMI. J Nutr $141,2219-25$ (2011). 
7. Corella, D. et al. Statistical and biological gene-lifestyle interactions of MC4R and FTO with diet and physical activity on obesity: new effects on alcohol consumption. PLoS One 7, e52344 (2012).

8. Graff, M. et al. Genome-wide physical activity interactions in adiposity - A metaanalysis of 200,452 adults. PLoS Genet 13, e1006528 (2017).

9. Kilpelainen, T.O. et al. Physical activity attenuates the influence of FTO variants on obesity risk: a meta-analysis of 218,166 adults and 19,268 children. PLoS Med 8, e1001116 (2011).

10. Moore, R. et al. A linear mixed-model approach to study multivariate geneenvironment interactions. Nat Genet 51, 180-186 (2019).

11. Phillips, C.M. et al. High dietary saturated fat intake accentuates obesity risk associated with the fat mass and obesity-associated gene in adults. J Nutr 142 , 824-31 (2012).

12. Qi, Q. et al. Dietary Intake, FTO Genetic Variants, and Adiposity: A Combined Analysis of Over 16,000 Children and Adolescents. Diabetes 64, 2467-76 (2015).

13. Qi, Q. et al. FTO genetic variants, dietary intake and body mass index: insights from 177,330 individuals. Hum Mol Genet 23, 6961-72 (2014).

14. Tyrrell, J. et al. Gene-obesogenic environment interactions in the UK Biobank study. Int J Epidemiol 46, 559-575 (2017).

15. Young, A.I., Wauthier, F. \& Donnelly, P. Multiple novel gene-by-environment interactions modify the effect of FTO variants on body mass index. Nat Commun 7, 12724 (2016).

16. Smith, P.G. \& Day, N.E. The design of case-control studies: the influence of confounding and interaction effects. Int J Epidemiol 13, 356-65 (1984).

17. Thomas, D. Gene--environment-wide association studies: emerging approaches. Nat Rev Genet 11, 259-72 (2010).

18. Bourgon, R., Gentleman, R. \& Huber, W. Independent filtering increases detection power for high-throughput experiments. Proc Natl Acad Sci U S A 107, 9546-51 (2010).

19. Liley, J. \& Wallace, C. Accurate error control in high dimensional association testing using conditional false discovery rates. bioRxiv (2019).

20. Andreassen, O.A. et al. Improved detection of common variants associated with schizophrenia and bipolar disorder using pleiotropy-informed conditional false discovery rate. PLoS Genet 9, e1003455 (2013).

21. Liley, J. \& Wallace, C. A pleiotropy-informed Bayesian false discovery rate adapted to a shared control design finds new disease associations from GWAS summary statistics. PLoS Genet 11, e1004926 (2015).

22. Genomes Project, C. et al. A global reference for human genetic variation. Nature 526, 68-74 (2015).

23. Benjamini, Y. \& Hochberg, Y. Controlling the false discovery rate: a practical and powerful approach to multiple testing. Journal of the royal statistical society. Series B (Methodological), 289-300 (1995).

24. Taylor, J. \& Tibshirani, R. A tail strength measure for assessing the overall univariate significance in a dataset. Biostatistics 7, 167-81 (2006).

25. Pers, T.H., Timshel, P. \& Hirschhorn, J.N. SNPsnap: a Web-based tool for identification and annotation of matched SNPs. Bioinformatics 31, 418-20 (2015).

26. Storey, J.D. A direct approach to false discovery rates. Journal of the Royal Statistical Society: Series B (Statistical Methodology) 64, 479-498 (2002).

27. Storey, J.D. \& Tibshirani, R. Statistical significance for genomewide studies. Proc Natl Acad Sci U S A 100, 9440-5 (2003).

28. Speliotes, E.K. et al. Association analyses of 249,796 individuals reveal 18 new loci associated with body mass index. Nat Genet 42, 937-48 (2010). 
29. Akiyama, M. et al. Genome-wide association study identifies 112 new loci for body mass index in the Japanese population. Nat Genet 49, 1458-1467 (2017).

30. Berndt, S.I. et al. Genome-wide meta-analysis identifies 11 new loci for anthropometric traits and provides insights into genetic architecture. Nat Genet 45, 501-12 (2013).

31. Fox, C.S. et al. Genome-wide association for abdominal subcutaneous and visceral adipose reveals a novel locus for visceral fat in women. PLoS Genet 8 , e1002695 (2012).

32. Riveros-McKay, F. et al. Genetic architecture of human thinness compared to severe obesity. PLoS Genet 15, e1007603 (2019).

33. Klimentidis, Y.C. et al. Genome-wide association study of habitual physical activity in over 377,000 UK Biobank participants identifies multiple variants including CADM2 and APOE. Int J Obes (Lond) 42, 1161-1176 (2018).

34. Buniello, A. et al. The NHGRI-EBI GWAS Catalog of published genome-wide association studies, targeted arrays and summary statistics 2019. Nucleic Acids Res 47, D1005-D1012 (2019).

35. Morris, J. et al. Genetic variation in CADM2 as a link between psychological traits and obesity. Sci Rep 9, 7339 (2019).

36. Ahmad, S. et al. Gene x physical activity interactions in obesity: combined analysis of 111,421 individuals of European ancestry. PLoS Genet 9, e1003607 (2013).

37. Richardson, A.S. et al. Moderate to vigorous physical activity interactions with genetic variants and body mass index in a large US ethnically diverse cohort. Pediatr Obes 9, e35-46 (2014).

38. Rask-Andersen, M., Karlsson, T., Ek, W.E. \& Johansson, A. Genome-wide association study of body fat distribution identifies adiposity loci and sexspecific genetic effects. Nat Commun 10, 339 (2019).

39. Kichaev, G. et al. Leveraging Polygenic Functional Enrichment to Improve GWAS Power. Am J Hum Genet 104, 65-75 (2019).

40. Locke, A.E. et al. Genetic studies of body mass index yield new insights for obesity biology. Nature 518, 197-206 (2015).

41. Felix, J.F. et al. Genome-wide association analysis identifies three new susceptibility loci for childhood body mass index. Hum Mol Genet 25, 389-403 (2016).

42. Karlsson Linner, R. et al. Genome-wide association analyses of risk tolerance and risky behaviors in over 1 million individuals identify hundreds of loci and shared genetic influences. Nat Genet 51, 245-257 (2019).

43. Dai, J.Y., Kooperberg, C., Leblanc, M. \& Prentice, R.L. Two-stage testing procedures with independent filtering for genome-wide gene-environment interaction. Biometrika 99, 929-944 (2012).

44. Casale, F.P., Rakitsch, B., Lippert, C. \& Stegle, O. Efficient set tests for the genetic analysis of correlated traits. Nat Methods 12, 755-8 (2015).

45. Loh, P.R. et al. Efficient Bayesian mixed-model analysis increases association power in large cohorts. Nat Genet 47, 284-90 (2015).

46. Casale, F.P., Horta, D., Rakitsch, B. \& Stegle, O. Joint genetic analysis using variant sets reveals polygenic gene-context interactions. PLoS Genet 13, e1006693 (2017).

47. Bycroft, C. et al. The UK Biobank resource with deep phenotyping and genomic data. Nature 562, 203-209 (2018).

48. Fesinmeyer, M.D. et al. Genetic risk factors for BMI and obesity in an ethnically diverse population: results from the population architecture using genomics and epidemiology (PAGE) study. Obesity (Silver Spring) 21, 835-46 (2013).

49. Abraham, G., Qiu, Y. \& Inouye, M. FlashPCA2: principal component analysis of Biobank-scale genotype datasets. Bioinformatics 33, 2776-2778 (2017). 